Article

\title{
Wireless Sensor Network Aided Assembly Line Monitoring According to Expectations of Industry 4.0
}

\author{
László Gogolák ${ }^{1, *}$ and Igor Fürstner ${ }^{2}$ \\ 1 Technical Department, Faculty of Engineering, University of Szeged, 6720 Szeged, Hungary \\ 2 Donát Bánki Faculty of Mechanical and Safety Engineering, Óbuda University, 1034 Budapest, Hungary; \\ furstner.igor@bgk.uni-obuda.hu \\ * Correspondence: gogolak@mk.u-szeged.hu
}

check for updates

Citation: Gogolák, L.; Fürstner, I

Wireless Sensor Network Aided Assembly Line Monitoring According to Expectations of Industry 4.0. Appl. Sci. 2021, 11, 25. https://doi.org/ 103390/app11010025

Received: 23 November 2020 Accepted: 18 December 2020 Published: 22 December 2020

Publisher's Note: MDPI stays neutral with regard to jurisdictional clai$\mathrm{ms}$ in published maps and institutional affiliations.

Copyright: (C) 2020 by the authors. Licensee MDPI, Basel, Switzerland. This article is an open access article distributed under the terms and conditions of the Creative Commons Attribution (CC BY) license (https:// creativecommons.org/licenses/by/ $4.0 /)$.

\begin{abstract}
Striving for excellence during the assembling process through incorporating the expectations of Industry 4.0 requires complex information management on issues of overall system status, especially the physical characteristics and position of the parts being assembled, as well as the assembling units and tools. This research introduces both an overall customized assembling system supervision model, which is based on a modified four-layer control system hierarchy that suits the specific requirements of such systems and the developed wireless sensor network technology for assembling process management with a particular focus on localization. The developed model highlights the localization problems of the system as well as other aspects required for overall system status determination. The localization of assembled parts is based on the fingerprint localization method by using the received signal strength indicator. The proposed localization algorithms are based either on artificial neural networks or on the weighted k-nearest neighbor method. The developed model has been tested both in laboratory conditions and in a simulated industrial environment. The research results offer a general solution to the problem of assembling system supervision, regardless of size and shape, with emphasis on the localization problem solution.
\end{abstract}

Keywords: assembling process monitoring; wireless sensor network; localization technology

\section{Introduction}

If a company strives to be a market leader, it must incorporate the newest research results into all its activities. Therefore, the production systems also have to be state of the art. Having in mind the assembling systems, which are part of the production systems, one of the aspects that requires special attention is the control of the assembling process. To be able to control the assembling process successfully, data is required about the status, physical characteristics, and position of the parts being assembled. Additionally, information on the status of the assembling units and tools is needed as well. Localization and tracking of the parts and subassemblies in the assembling system presents the biggest challenge to deal with.

The popularity of wireless technology in industrial applications was well predicted in the research of Hatler [1]. The relatively low price and easy implementation of wireless technology indicated its use for assembling process supervision. Therefore, extensive research has been done regarding the use of wireless technology in the related technology [2]. One of the possible implementations was the multi agent systems, but this approach did not have adequate success in automation applications [3-6].

Regarding the control and data acquisition applications in industrial systems, the supervisory control and data acquisition-SCADA systems are used [7]. Additionally, applications for diagnostics, testing, and control are also used, but the communication is usually based on Ethernet TCP/IP, RS-232, RS-485, and RS-422 communication protocols [8]. Distributed automation architecture, as well as IoT systems, require state-of-the-art communication technologies to meet the requirements of Industry 4.0. [9-12]. 
Currently, wireless communication is not within the first ten most popular communication protocols. Sensors and actuators are connected to the control unit, which is usually a programmable logic controller-PLC. This use of sensors connected by wires to the control unit carries high installation costs and is highly inflexible if a reconfiguration is needed.

A standard method for designing assembling and production systems by using wireless technology already exists [13]. In the case of production systems, the literature review defines "Wireless Manufacturing" [14]. In this case, part of the information is transferred by wireless technology. The success of using wireless technology is evident in the case of using radio-frequency identification-RFID technology. RFID technology is commonly used for controlling the material and part flows in production and storing [15,16]. The literature review also presents the implementation of wireless technology in tracking the material and part flows in assembling systems $[17,18]$, but the developed solutions are specific for a given assembling task; therefore, an overall solution is yet to be presented. The overall solution in this field of research requires the fusion of research results of different technical fields (electronics, sensor techniques, communication technologies, imbedded systems, etc.).

Recent development results in the field of electronics resulted in component miniaturization, low energy consumption, and higher levels of reliability $[19,20]$. In the field of sensor techniques, the miniaturization, the level of precision, less noise dependency, low energy consumption, and wider spectrum of measurable non-electric signals is possible. The communication technologies allow new communication protocols [9], which are reliable in high noise-level surroundings, and also low energy consumption in the case of wireless communication. New microcontrollers allow for higher number and quality of A/D and D/A converters, etc. Additionally, the computing speed, energy efficiency as well as the operational memory are higher and bigger, allowing the usage of microcontrollers in several embedded systems [21]. This points towards the presented research, which is to incorporate these technologies to the assembling systems, i.e., in the supervision of the assembling systems.

The paper aims at contributing to fill in a gap in literature by introducing the necessary methodology and elements, which will result in a successful supervision of the assembling processes, by using wireless technology. Therefore, the main objective of the paper is to develop an overall customized supervision model that fits the requirements of the assembling systems, and to incorporate wireless sensor network (WSN) technology for assembling process management, i.e., for solving localization problems during the assembling process. The main objective points towards the definition of several sub-objectives:

- Define the necessary technology and equipment for the supervision process;

- Define the usability, i.e., the limitations of wireless technology, by taking into account the requirements for controlling the assembly processes;

- Define the supervision algorithms;

- Adapt the general supervision model structure to be able to respond adequately to the specific requirements of managing the process of assembling by using wireless sensor network (WSN) technology;

- Define the characteristics of the identified elements of the adapted general control system;

- Establish the connection between the system elements.

From a methodological standpoint, the proposed solution is based on knowledge gained from the literature that deals with supervising the assembling process, the literature that deals with implementing WSN technology, the analysis of existing solutions, and the authors' experience in incorporating WSN technology in production processes [22].

\section{Materials and Methods}

\subsection{Assembly Line Supervision Model Based on WSN Technology}

An Assembly line supervision system based on WSN technology has to be developed according to expectations of standard control systems models [22]. The general supervision 
model [23] is adapted to be able to respond adequately to the specific requirements of managing the process of assembling by using WSN technology. The proposed WSN supervision model is presented in Figure 1.

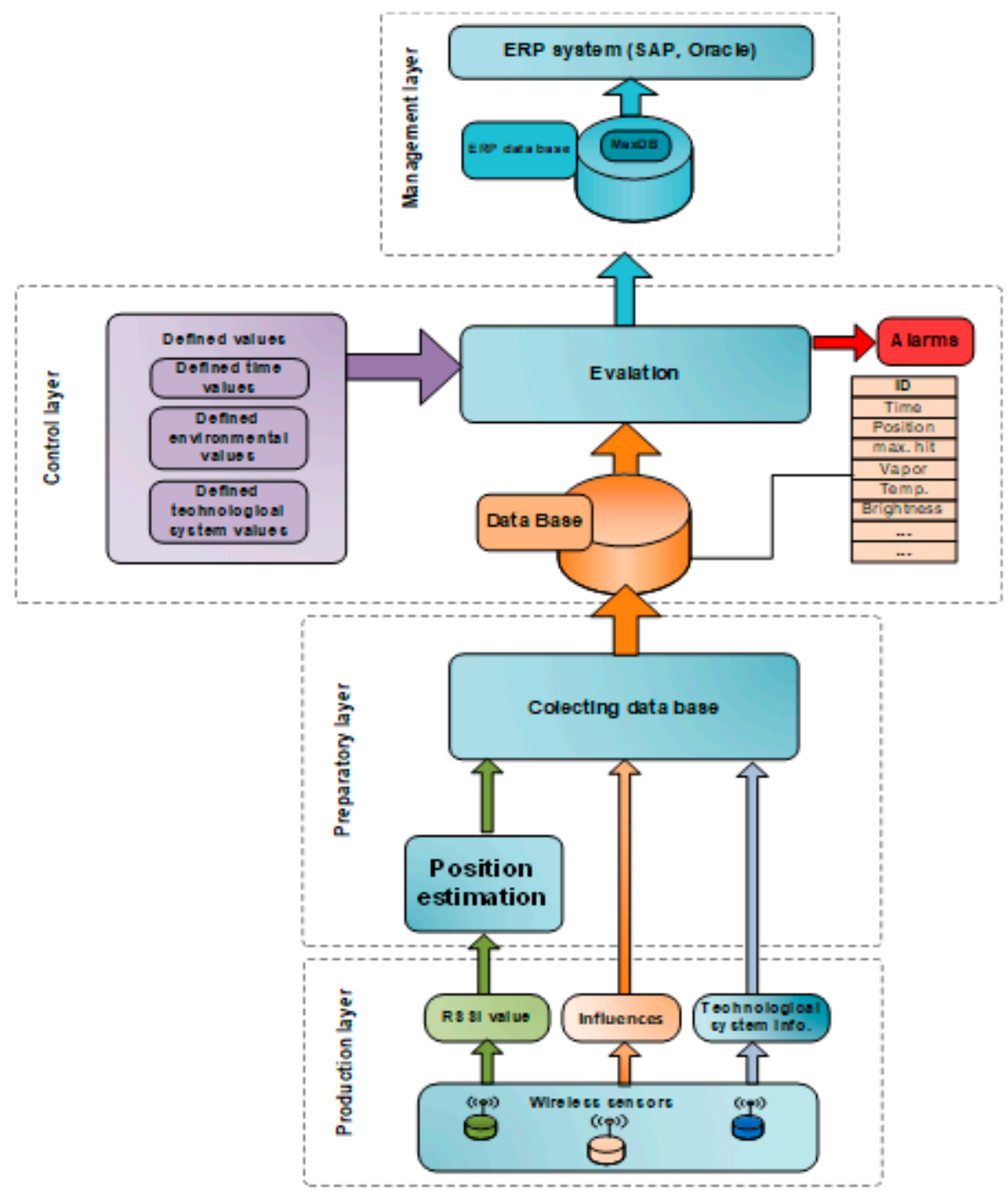

Figure 1. The proposed adapted WSN supervision system model.

The WSN supervisor model can be divided into four layers, the "Production", "Preparatory", "Control", and "Management" layers. If compared with the general model [23], several differences can be noticed. The layer of "Sensors and actuators" is divided into two layers, the "Production" and the "Preparatory" layer, while the "Production control" and the ERP layer are joined in the "Management" layer. The need for such a deviation from the general model is caused by the specificity of the WSN control system that is proposed.

The "Production" layer includes the wireless sensors, which are implemented in the assembling system. Depending on their specific role in the monitoring process, four types of sensors can be defined:

- Wireless sensors, which are mounted on the base part (base sensors);

- Auxiliary wireless modules, which are used to determine the base part's position (anchors);

- Wireless sensors, which are mounted on the technological system (technology sensors);

- Wireless base modules for collecting data (collecting sensors).

The highest number of sensors is attached to the base part of the assembled product. The detailed structure of the wireless sensors in this layer is presented in Figure 2. 


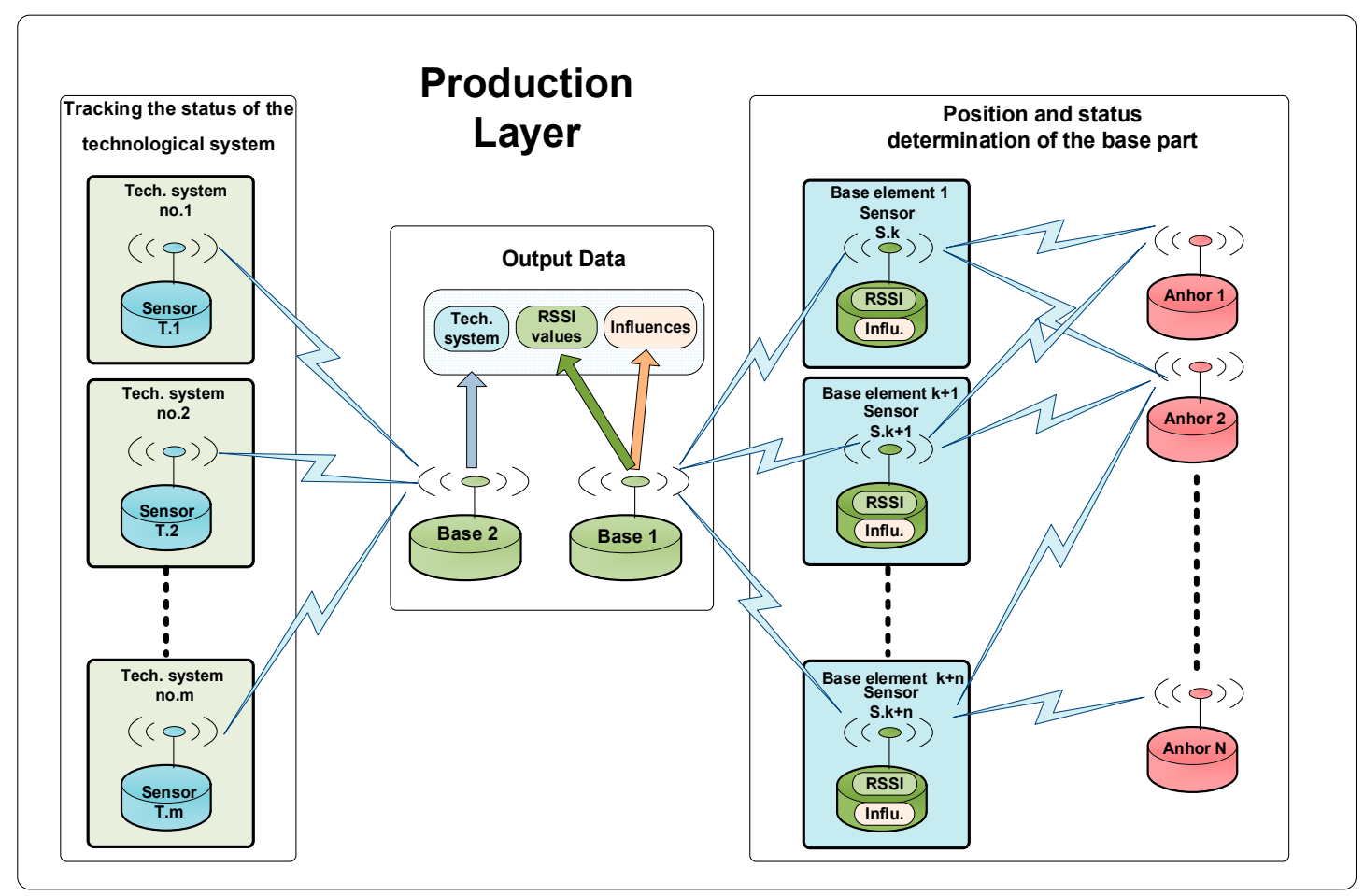

Figure 2. The "Production" layer.

The auxiliary anchor wireless modules, which have a fixed known position, are used for determining the position of different base parts at the assembling line. The status of the technological system is determined by separate sensors, which are mounted directly at the required positions inside the assembling system. The wireless base modules for collecting data are used to collect data from all wireless sensors. There is a separate module for collecting data from the base parts and a separate module for collecting data from the technological system. Detailed description of the production layer can be found in [22].

The "Preparatory" layer provides for the data transfer, which is done by wired communication via standard communication protocols. In this layer, one or more industrial computers are placed. Their three main tasks are to continuously run the localization algorithms presented in Section 2.5, which determines the actual position of the base parts based on measured received signal strength indicator values (RSSI), to unify the data and to form a database, which is stored in the "Control" layer.

The industrial computer is usually physically placed close to the assembly line, due to communication restrictions of the collecting sensors. The communication between the "Preparatory" and upper layers is done either by wire (LAN) or by wireless (WLAN) communication.

The "Control" layer evaluates the measured, i.e., the determined values. The database stores all data that is collected in the "Production" layer. The evaluation is done by comparing the measured data with the data defined in the assembly design phase. Based on the comparison, the appropriate decision about the assembly process is generated.

The evaluation of the assembly system is performed on three levels:

- Evaluation of the balancing quality of the assembly system.

- Evaluation of the quality of the assembly process based on measured physical influences.

- Evaluation of the technological systems' status.

At each level, a separate report is generated. Reports contain their evaluation decisions, which are stored in the database of the "Management" layer. If the report contains critical information about the quality of the assembly system or process, it is sent to an appropriate person. 
The management layer performs the global analyses of the quality and effectiveness of the assembly system and process.

The proposed supervision model presents the basis for the implementation of the proposed localization technology, which represents the most complex part of the control process within the assembly system.

\subsection{Usability of WSN Technology during Product Assembling}

Sensor networks contain several wireless sensing devices, which communicate via wireless technology. This communication is usually performed by IEEE 802.15.4 standard, which is also known as Zigbee technology. This communication protocol works at $2.4 \mathrm{GHz}$, and the communication is point-to-point.

The structure of the assembly line can be described as a series of work stations at which the assembling process is performed [17]. Along the assembly line, the assembling process runs sequentially (Figure 3).

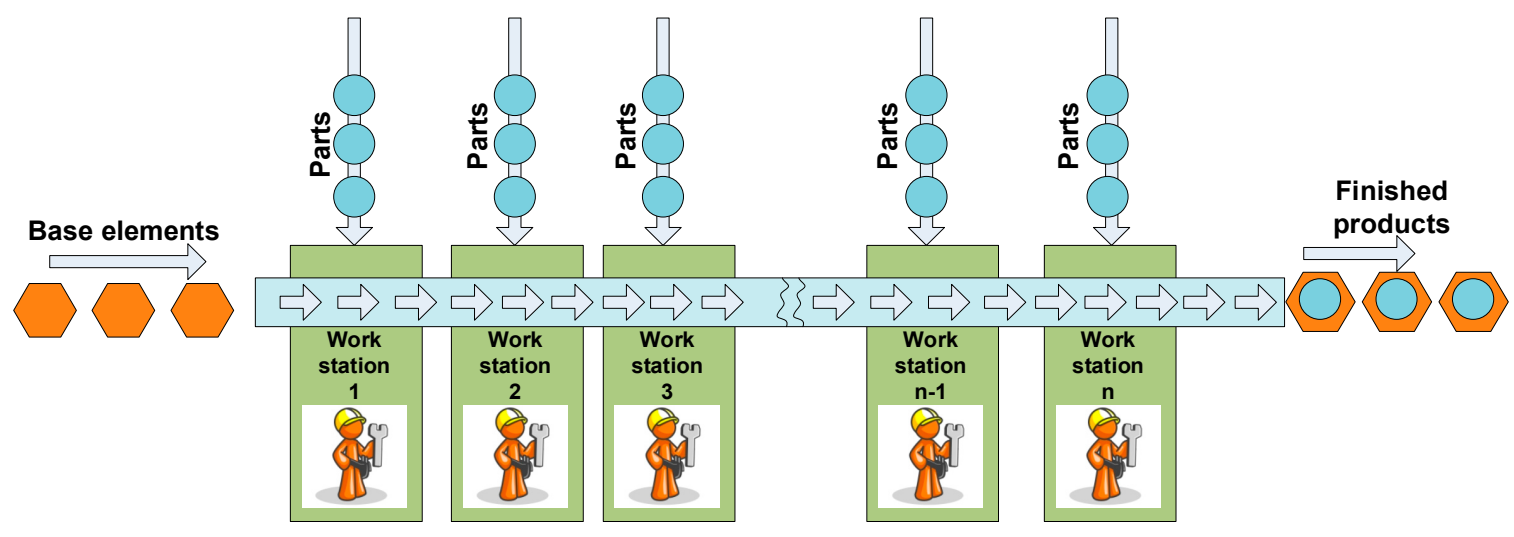

Figure 3. Assembly line structure example.

At first workstation, the base part is placed, usually in equal time intervals. After the last workstation, the product is fully assembled. To be able to use WSN technology for monitoring purposes, the sensor has to be mounted on the base part of the assembly. Therefore, the usability of WSN technology largely depends on the product itself, i.e., on the overall dimensions of the product. Due to the fact that nowadays wireless sensors are active instruments, i.e., they have their own power supply, their own dimensions are relatively large relative to, for example, RFID tags. Therefore, one of the criteria for implementing WSN is that the base part should be larger than the sensor. Today, the smallest available sensor modules have a diameter of $25 \mathrm{~mm}$ (e.g., "Mica2 dot") [22]. Generally, for appropriate use of WSN, the base part has to be twice the size of the sensor.

During the research, Crossbow Iris wireless node was used for the proposed model. It uses $2.4 \mathrm{GHz}$ frequency using the IEE 802.15.4 standard and is using the Zigbee communication protocol stack [22]. The Iris sensor nod can be used for different applications, because it has a 51-pin connector that allows for expansion of the base with different sensor units, i.e., temperature sensor, photo sensor, microphone, accelerometer, magnetic sensor, humidity sensor, pressure sensor, etc.

The sensor, which is mounted on the base part of the product, must not interfere with the defined assembling procedures performed either by humans or by manipulators and robots. Therefore, part lists and other documentation prepared for the assembling process must be thoroughly analyzed to be able to define the right position for mounting the sensor at the first workstation.

By this, the sensor becomes an integral part of the product during the assembling process. At the end of the process, the sensor has to be un-mounted. Therefore, not only the mounting procedure, but also the un-mounting one has to be defined to be as simple and fast as possible, for minimizing the influence on the assembling procedure. Another 
possibility is to integrate the wireless sensor module into the base part holder. By this the mounting and un-mounting of the sensor are avoided, but the holder becomes more complex.

\subsection{Parameters That Influence the Localization Procedure and the Quality of the Assembling Process}

Having a mounted wireless sensor on the base part allows the monitoring of the assembling process, i.e., the position monitoring of the base part in the assembling system. The usage of WSN technology raises some questions due the industrial environment of the assembling systems. The interference, diffraction, and dissipation of the wireless signals in industrial environment can be critical and therefore ask for additional attention during the design of the WSN.

To be able to localize the mobile sensor mounted on the base part, several referentfixed sensors, i.e., anchor sensors, are needed as well. By optimally positioning the anchor sensors, the unwanted effects of the industrial environment can be minimized.

The localization is realized based on predefined resolution. This resolution is also used when the RSSI map of the assembly line is recorded. The localization precision, but also the complexity of the system, is higher if the resolution is higher. Therefore the resolution has to be optimized for every assembling system individually. During the development of the localization system, to be able to define the optimal resolution of the localization system, several characteristics of the system have to be analyzed:

- Special requirements regarding the accuracy of localization

- The overall dimensions of the assembling line (lengths and width)

- The number of workstations in the assembling line

- The number of used anchors

- The overall dimensions of the product

- Available hardware and software resources implemented into the localization system

- Specific requirements of the assembling system

- Technical specification of the wireless modules.

Every assembling line has a defined working environment, which is necessary to have a quality product at the end of the assembling process. Usually, the quality of the product is analyzed at the end of the process, not during the process. Several physical parameters could influence the product during the process [18].

WSN technology allows for monitoring the quality of the product during the whole assembling process. Not only the position of the product can be tracked in time and space, but also a number of physical parameters can be checked if required as well:

- The temperature of the assembly

- The relative humidity of the surrounding air

- The illumination of the assembly

- The unwanted acceleration of the assembly

- The sound level around the assembly

Having in mind the specificities of the assembling system, it can be concluded that the monitoring of physical parameters has to be performed not only on the assembly itself, but also independently on each workstation and on each technological system, e.g., the screwing system, the gluing system, the riveting system, etc. For that, separate wireless sensors could be mounted directly on each workstation and on used technological systems.

\subsection{Localization Technologies}

Localization technologies are either based on satellite technology or on network structures. The localization technologies in closed spaces, such as in assembling facilities, require the use of network structures. Usually, these technologies require the determination of the distance between the nodes. To determine the position of the moving node, the distance between the moving node and static nodes, which are usually at fixed positions 
(anchor nods or access points), is determined. To be able to determine the distance between the nodes, different methods can be used:

- Methods based on Time of Arrival [24]

- Methods based on Time Difference of Arrival [24]

- Methods based on the Angle of Arrival [24]

- Methods based on Received Signal Strength Indicator (RSSI) $[25,26]$

As opposed to the first three methods, which do not have the specified medium for localization, the RSSI method uses wireless technology. RSSI localization is based on using the strength of the signal between two nodes for position determination. The RSSI method is not limited by a specific wireless communication standard. Therefore, WiFi (WLAN), Bluetooth, and ZigBee wireless technologies can be used as well. A huge advantage of RSSI method is the fact that no additional hardware is required for the localization. Wireless nodes use radio modules for wireless communication, which can be also used for RSSI value determination for neighboring nodes. This simple structure enables for low energy consumption, small dimensions, and low costs.

Fingerprint localization method uses RSSI values obtained by wireless communication between sensor nodes [27]. Mostly, it is used for closed space localization by using WSN. The method requires fixed-anchor nodes. Fingerprint localization is performed in two steps: offline and online [28].

During offline determination, the so-called RSSI map is formed for the chosen space and environment. The RSSI map is determined by recording the RSSI values between the mobile and anchor nodes, i.e., anchors, which are positioned at known fixed positions. The number and location of the anchors is determined based on the characteristics of the space and surroundings and the precision requirements. The mobile node is moved from one known position to another known position and the RSSI value is recorded at all positions (Figure 4). The known positions are usually set in a matrix structure. The resolution of the matrix, i.e., the distribution density of the positions is higher for higher accuracy requirements.

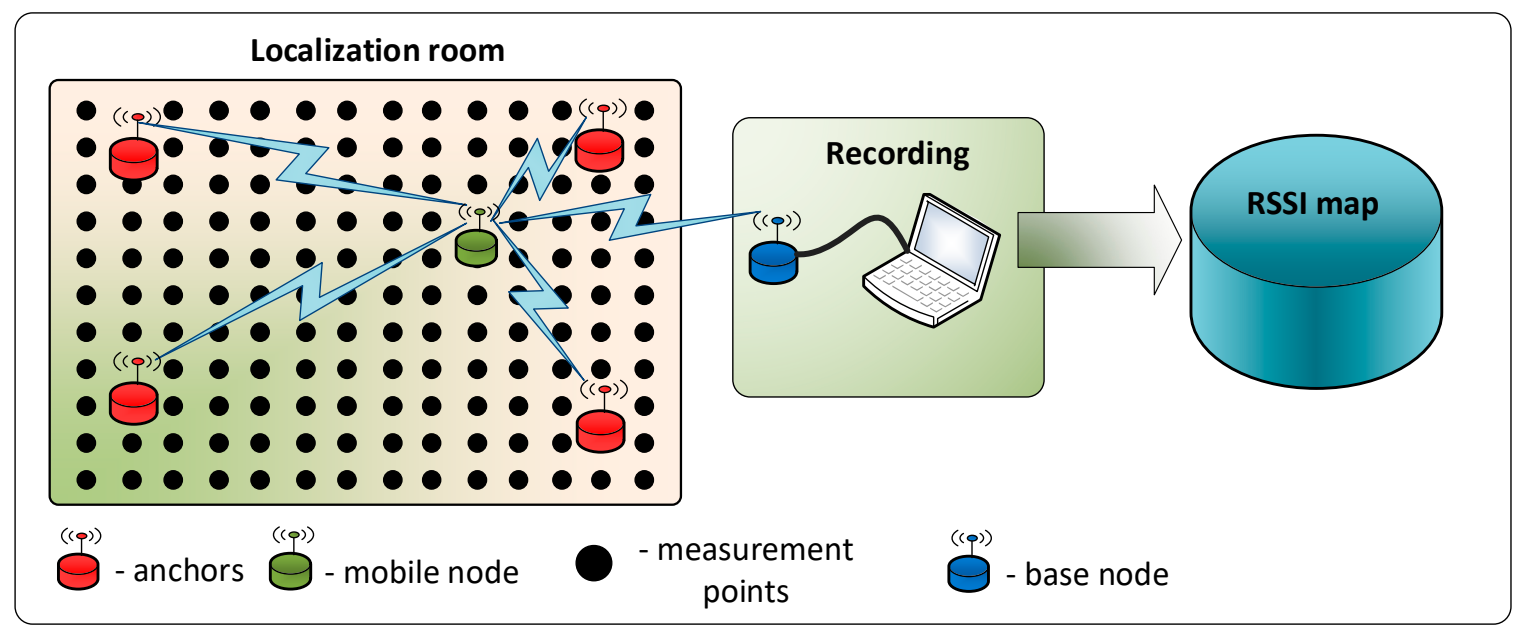

Figure 4. Offline phase of the fingerprint localization.

The recorded RSSI values are stored together with the position coordinates regarding to all anchors. The mobile nod sends a separate signal to all anchors. Based on the reply, the RSSI value is determined. The determined values are sent to a base node that is connected to the computer on which the data is stored.

During online localization, the mobile node is at an unknown position, which has to be determined. The mobile node sends signals to anchor nodes (Figure 5). 


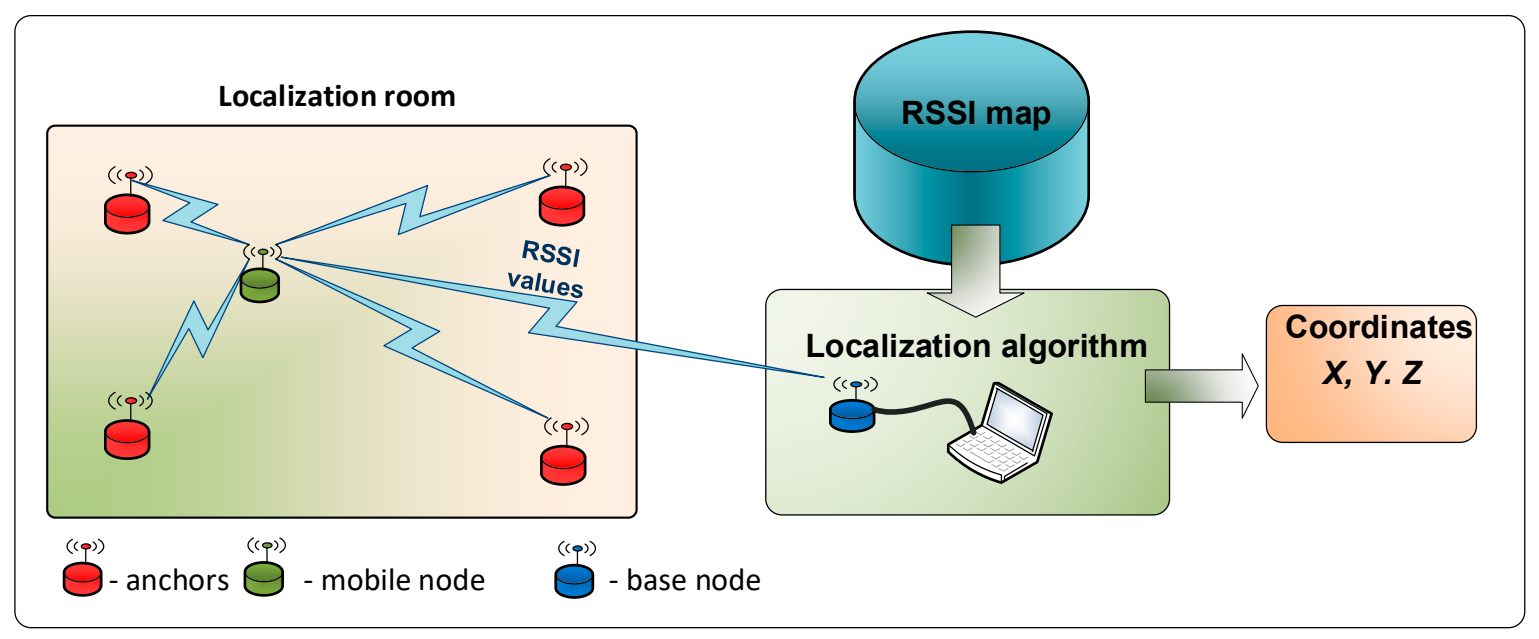

Figure 5. Online phase of the fingerprint localization.

Based on the reply from the anchors, the RSSI value is determined and sent to the base node, which transfers the data to the computer. Using localization algorithms, the unknown position is determined based on stored data from offline localization and data from online localization.

\subsection{Localization Algorithms}

To be able to successfully determine the location of the mobile node using fingerprint localization, a localization algorithm is to be used. The choice and application of the localization algorithm depends on the required information and has the biggest influence on the result [29]. Some algorithms are based on simple searching for similarities by comparing the measured RSSI data with the data from the data sample database. Usually, the successfully implemented algorithms are those used for classification purposes. In the proposed control model, two algorithms are used, Artificial Neural Networks (ANN) [30] and the weighted $k$-nearest neighbor algorithm (WkNN) [31].

The proposed ANN to be used in the control model is a multilayer one, which consists of the input and output layers and the internal layer as well [26,28]. At the output layer, the linear activation function is used, while at the internal layer, the hyperbolic tangent activation function is used. The proposed model uses the "Levenberg-Marquardt" training method.

The inputs to the ANN are the measured RSSI values for every anchor [31]. The number of the inputs to the ANN is determined by the number of anchors. At the output from the ANN, the required data form is the position coordinate of the mobile node. The training of the ANN was done by using the RSSI values measured in off-line mode and stored in the database. The stored data is organized in a matrix form $S_{j i}$, where the $s_{j i}$ elements of the matrix determine the RSSI value for the $j$-th measured position and $i$-th anchor.

For the implementation the WkNN algorithm, the RSSI values measured in off-line mode are used. The RSSI values measured in on-line mode at a certain moment of time $m$ are organized in a matrix form $S_{m i}$, where the $s_{m i}$ elements of the matrix determine the RSSI value for the $i$-th anchor.

Based on the measured $S_{m i}$, the similarity with one of the positions from $S_{j i}$ is determined by calculating the Euclidean distance $p_{j}$, where $n$ is the number of anchors:

$$
p_{j}=\sqrt{\sum_{i=1}^{n}\left(s_{m i}-s_{j i}\right)^{2}}
$$

The mostly similar position, i.e., referent point, is the point that has the minimal value for $p_{j}$. The WkNN method takes into consideration more than one point that is 
closest to the analyzed point (neighboring points). The number of neighboring points is $k$, which is the most important parameter to be used with this method and has to be defined at the beginning of the localization. The resulting coordinates of the mobile node are determined as:

$$
x y=\sum_{i=1}^{k} w_{i} x y_{i},
$$

where $w_{i}$ is a weight parameter, which is used to evaluate the potential neighboring points, while $x y_{i}$ are the coordinates of the potential points. The weight parameters are determined as:

$$
w_{j}=\frac{\frac{1}{p_{j}^{2}}}{\sum_{i=1}^{k} \frac{1}{p_{i}^{2}}}
$$

The testing results of the presented localization techniques can be found in paper [28], where indoor localization has been implemented in laboratory conditions based on RSSI. Here, the algorithms are applied in industrial environment.

\section{Results}

This chapter presents the test-laboratory setup, the determination, and the distribution of the initial off-line RSSI values of the anchors, and the test results and the comparison of the results accuracy in on-line mode, which are required for the assembly system evaluation.

\subsection{Laboratory Setup}

The industrial laboratory used for testing the proposed model is presented in Figure 6. It simulated the conditions of the real industrial surroundings. It consisted of an assembly line, which was set up for assembling a circulation pump. The assembly line consisted of professionally used elements, such as:

- Assembling workstations

- Conveyor with pallets for the base assembly

- Technological systems

- Industrial BOSCH-scara robot.

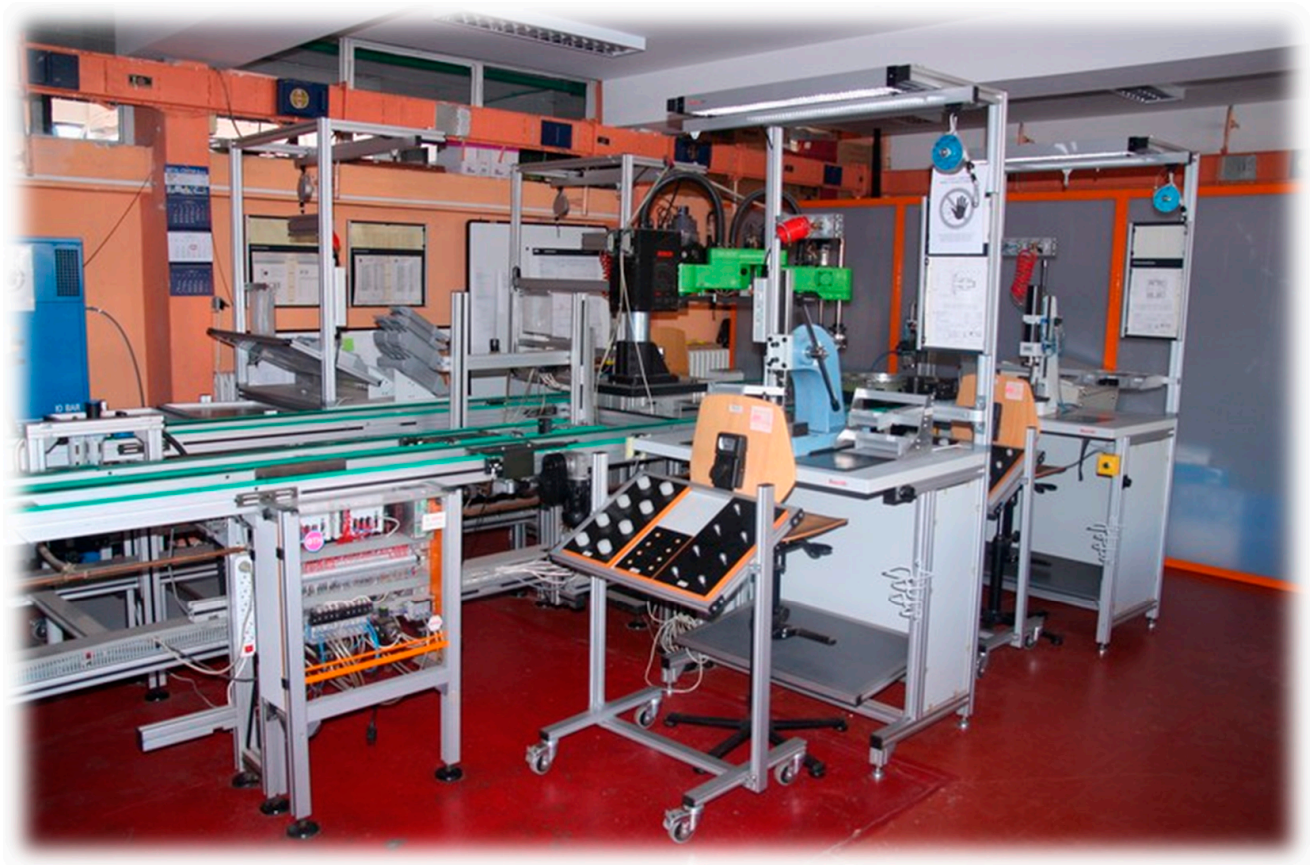

Figure 6. Industrial laboratory. 
By that, the assembly line created the surroundings that was similar to the real one.

Within the industrial laboratory, the wireless sensor network presented in Figure 7 was set up. Having in mind the specific characteristics of the assembling line, the proposed RSSI map for the system had to take into consideration the layout of the system. Fixed anchors were set-up too, which are presented as red dots.

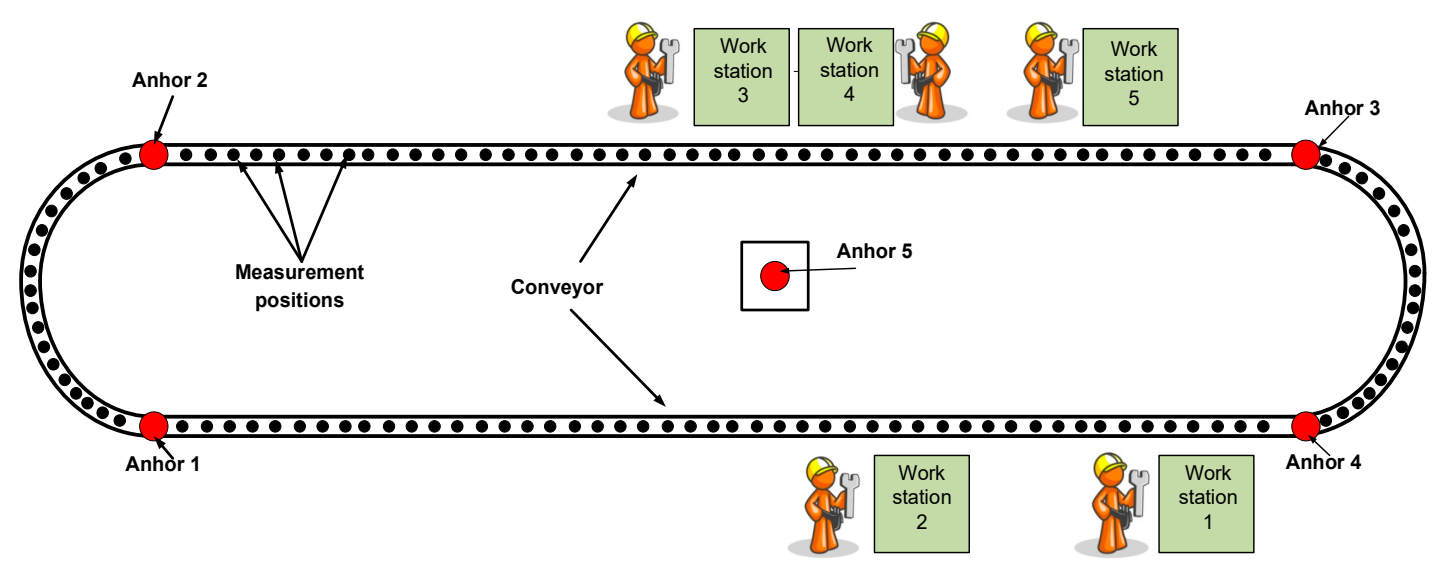

Figure 7. The structure of the measurement position and anchors at the industrial laboratory.

\subsection{Determination and Distribution of the Initial RSSI Values of the Anchors in Off-Line Mode}

For the purpose of obtaining the RSSI map, it was necessary to measure all the RSSI values between all anchors and measurement positions.

In the case of the industrial laboratory, the coordinates of the measurement positions were given in the form [segment, position]. The segment denotes the segment of the assembling line. The first segment was located between the anchors 1 and 2, the second segment was located between the anchors 2 and 3, etc. All positions were measured from the beginning of the segment in steps of $10 \mathrm{~cm}$ in length.

After obtaining the RSSI map values in of-line mode, various analyses were possible regarding RSSI in the function of the position and the structure of the anchors. One of the possibilities to make a satisfactory representation of the RSSI values distributions was to use MATLAB. The distribution was obtained by defining a color contour for every measured position.

The dimensions of the assembly line presented earlier determined 160 measurement points (the length of the line was $6 \mathrm{~m}$, while the width was $1.5 \mathrm{~m}$; therefore, along the length $2 \times 60$ measurement points were defined, while along the width $2 \times 20$ measurement points were defined). From Figure 7 one can conclude that the shorter sides of the assembly line had a shape of a half-circle, but for presentation purposes, the curved parts of the assembly line were approximated by straight lines. The industrial laboratory, as well as the assembling line itself, consisted of metal elements such as tools, holders, cables, etc.; therefore, in no circumstances could a homogeneous distribution be obtained.

In Figure 8, the distribution of the off-line RSSI values regarding different anchors is presented.

Results presented in Figure 8 are mainly used to check whether the anchors are positioned well. Based on the presented data, one can conclude that the anchors were positioned well, and that the number of the anchors was satisfactory for the required coverage of the assembling line.

The characteristic values for each anchor are given in Table 1. 


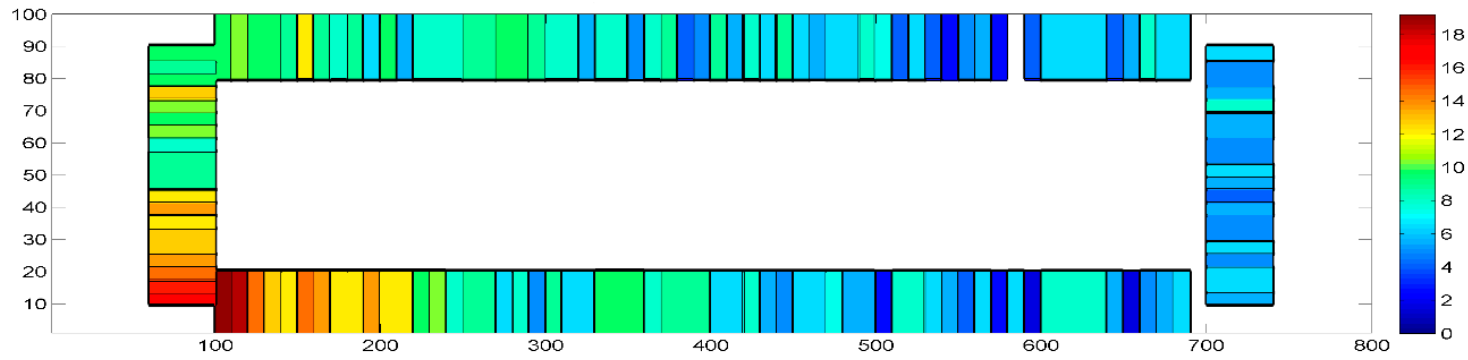

(a)

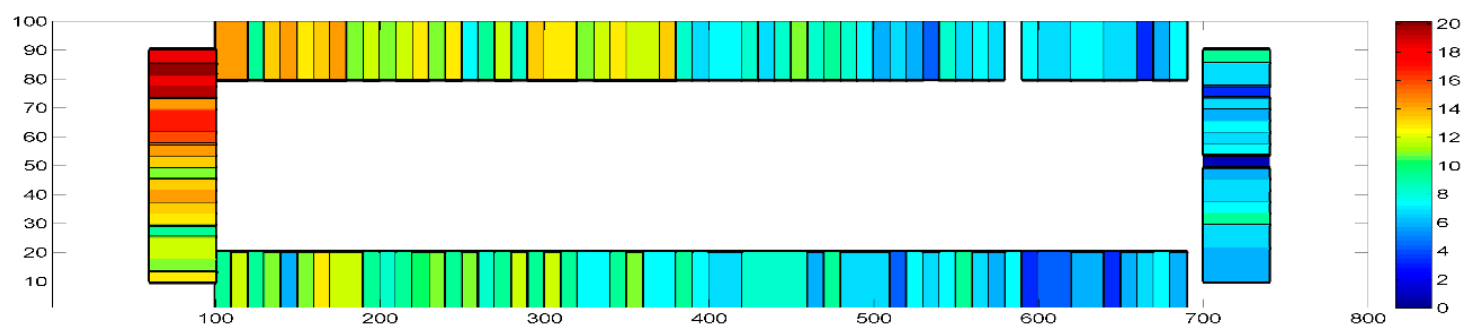

(b)

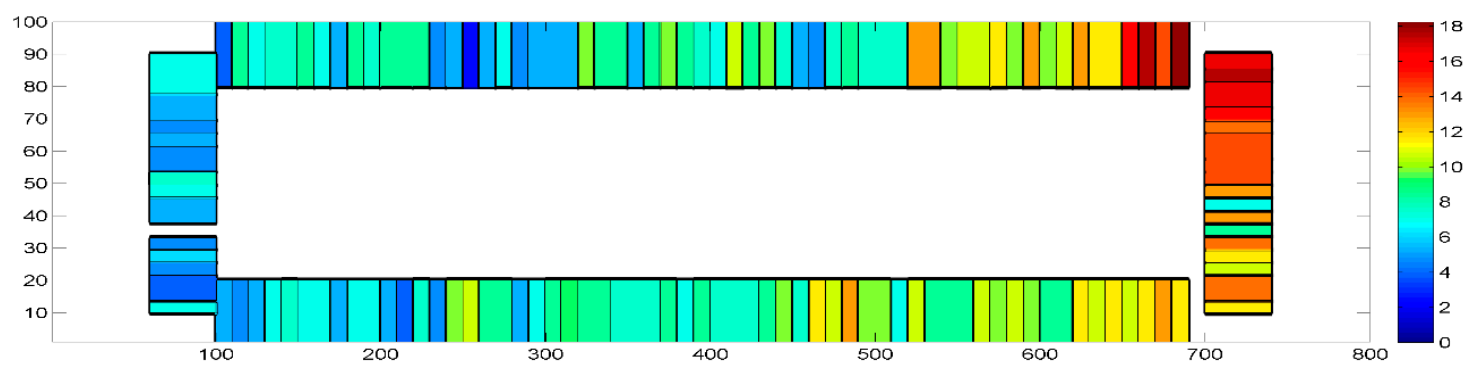

(c)

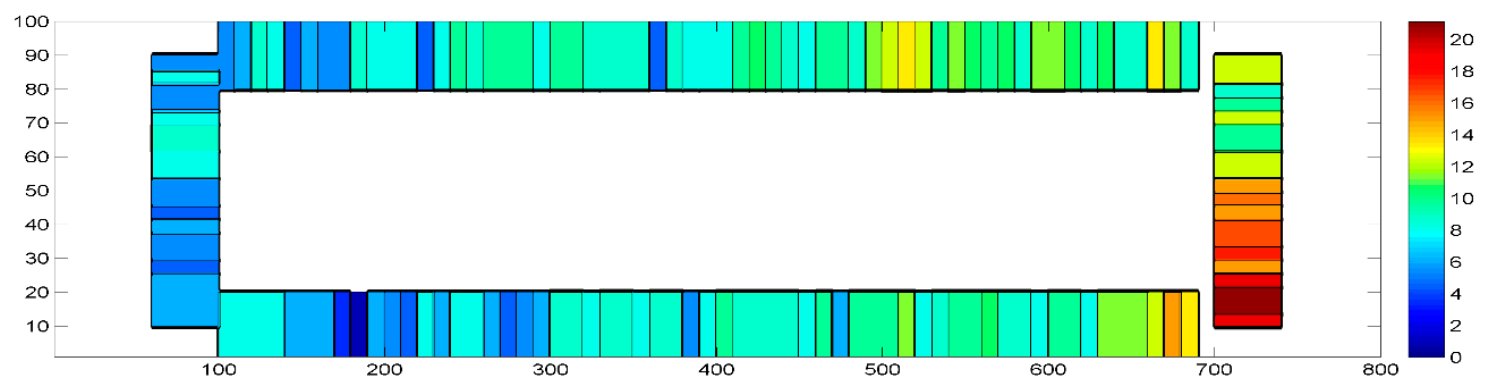

(d)

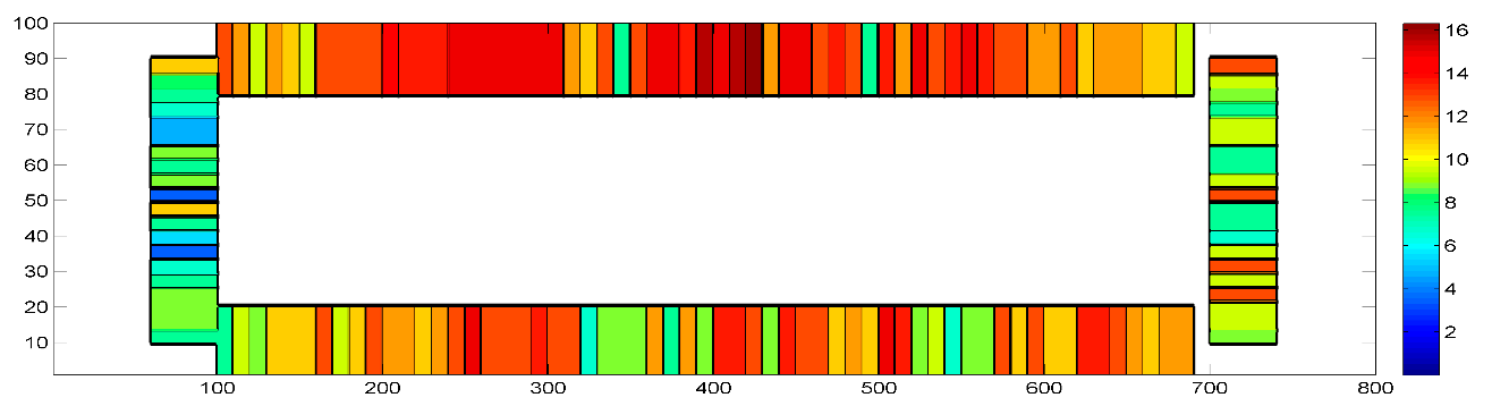

(e)

Figure 8. Distribution of the RSSI values for the Anchors: (a) Anchor 1; (b) Anchor 2; (c) Anchor 3; (d) Anchor 4; (e) Anchor 5. 
Table 1. Characteristic RSSI values in the case of industrial laboratory.

\begin{tabular}{ccccc}
\hline Anchor & Mean Value & Median & Min RSSI & Max RSSI \\
\hline 1 & 8.2764 & 8 & 0 & 20 \\
2 & 9.6335 & 9 & 0 & 21 \\
3 & 9.1894 & 9 & 0.5 & 20 \\
4 & 9.4317 & 9 & 1 & 22 \\
5 & 11.3882 & 12 & 3.4 & 17 \\
\hline
\end{tabular}

\subsection{Test Results and the Comparison of the Result Accuracy in On-Line Mode}

For testing purposes, the on-line gathered data was analyzed using both the neural networks and the WkNN method to determine the position of the wireless nodes. In this chapter, the localization results for test and industrial laboratories are compared having in mind both the off-line and on-line measurements. The results for the test laboratory were obtained earlier [28], but for some measurements, the results are shown together with the results obtained for the industrial laboratory, for easier and more quality comparison.

\subsubsection{Quality of the Localization Algorithms}

To be able to evaluate the assembly system, i.e., to compare the results obtained, the cumulative distribution function (CDF) was used. This function presents the probability of obtaining the certain localization error [32].

Firstly, the effectiveness of the localization is compared, for test samples from the RSSI values database (these are the points with "known" RSSI value). For this case, the following localization situations are compared:

- Localization at the industrial laboratory using neural networks (the number of neurons at the hidden layer is 200; the neurons are trained with the median database). The result is presented in Figure 9-curve IndusLab NN.

- Localization at the industrial laboratory using WkNN algorithms (the number of neighboring points is 3). The result is presented in Figure 9-curve IndusLab WkNN.

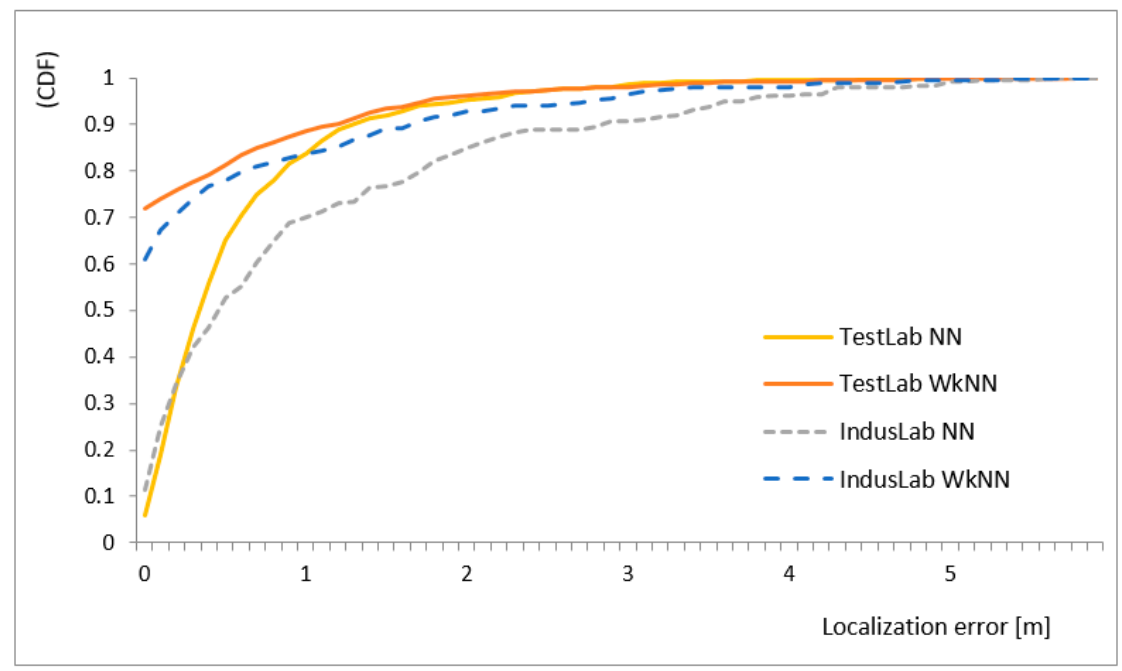

Figure 9. Comparison of localization accuracy for points with known RSSI values.

Following that, the quality of the localization algorithm was valued for test points, which had no RSSI values in the RSSI map (test points with unknown RSSI values). For this case, the following localization situations were compared:

- Localization at the industrial laboratory using neural networks (the number of neurons at the hidden layer is 200; the neurons are trained with the median database). The result is presented in Figure 10—curve IndusLab NN. 
- Localization at the industrial laboratory using WkNN algorithms (the number of neighboring points is 3). The result is presented in Figure 10-curve IndusLab WkNN.

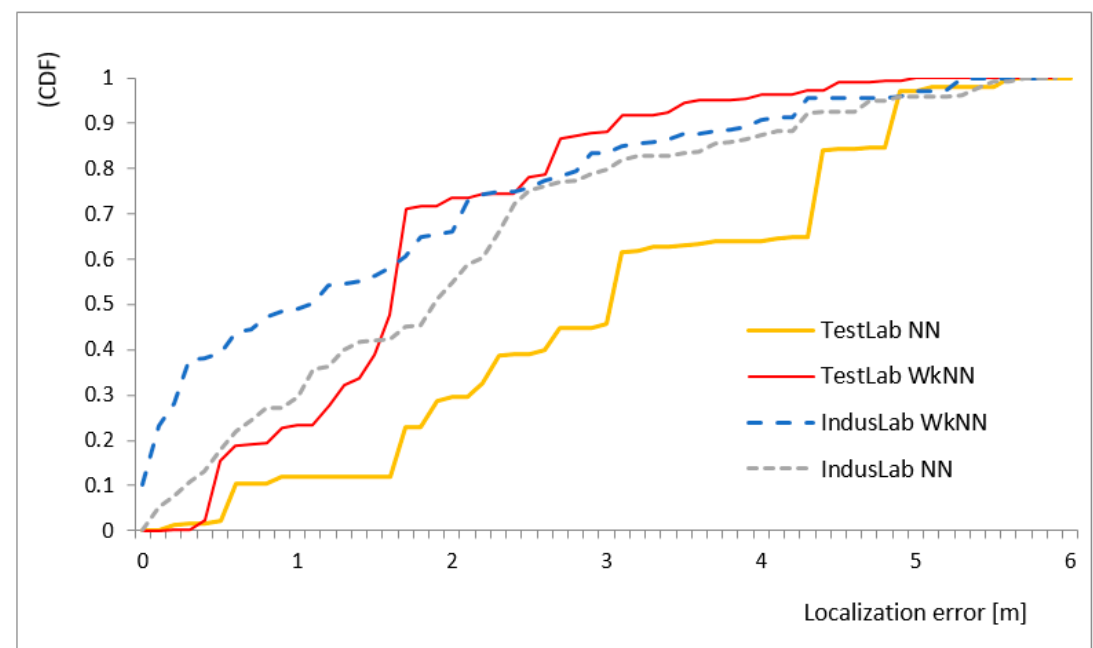

Figure 10. Comparison of localization accuracy for points with unknown RSSI values.

3.3.2. Influence of the Anchor Structure to the Quality of the Localization

Besides the influence of the localization algorithm, the quality of the localization is also influenced by the structure of anchor positioning. During research, five anchors were positioned for position determination of the moving anchor.

In the case of the industrial laboratory, the quality of the localization was tested by using five (all anchors), four (anchors 1, 2, 3, 4), and three anchors (anchors 1, 3, 5). The comparison of the localization quality for different number of anchors is presented in Figure 11.

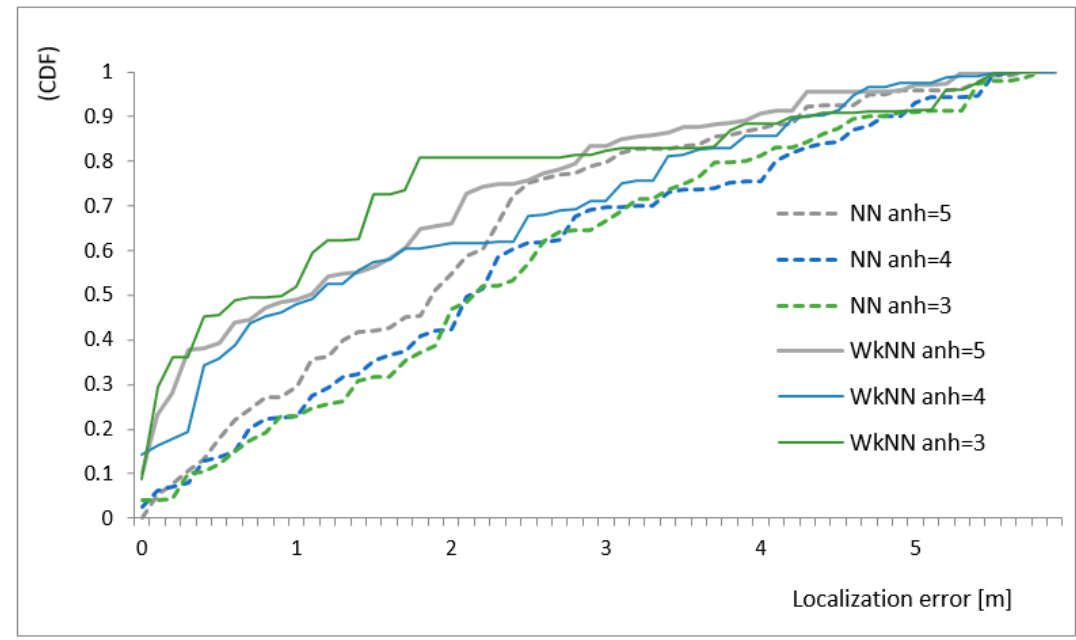

Figure 11. Comparison of the localization quality for different number of anchors for the industrial laboratory.

3.3.3. Influence of the Laboratory Structure to the Quality of the Localization

During the research, no specific factor or object was taken into consideration individually; however, several factors could influence the quality of the localization. The accuracy of the localization was determined at different segments of the laboratory instead. Based on the results of this analysis, the critical segments regarding the efficiency of the localization were determined. Based on this, insight was obtained regarding algorithm improvements and/or RSSI data filtering. 
In the case of the industrial laboratory, the highest influence was determined due to metal surfaces. In the reminder of this chapter, the segments with higher error values were analyzed. For the analysis, test samples with "known" RSSI values were used. In Figure 12, the localization error is presented if a neural network is used, for different numbers of neurons in the hidden layer.

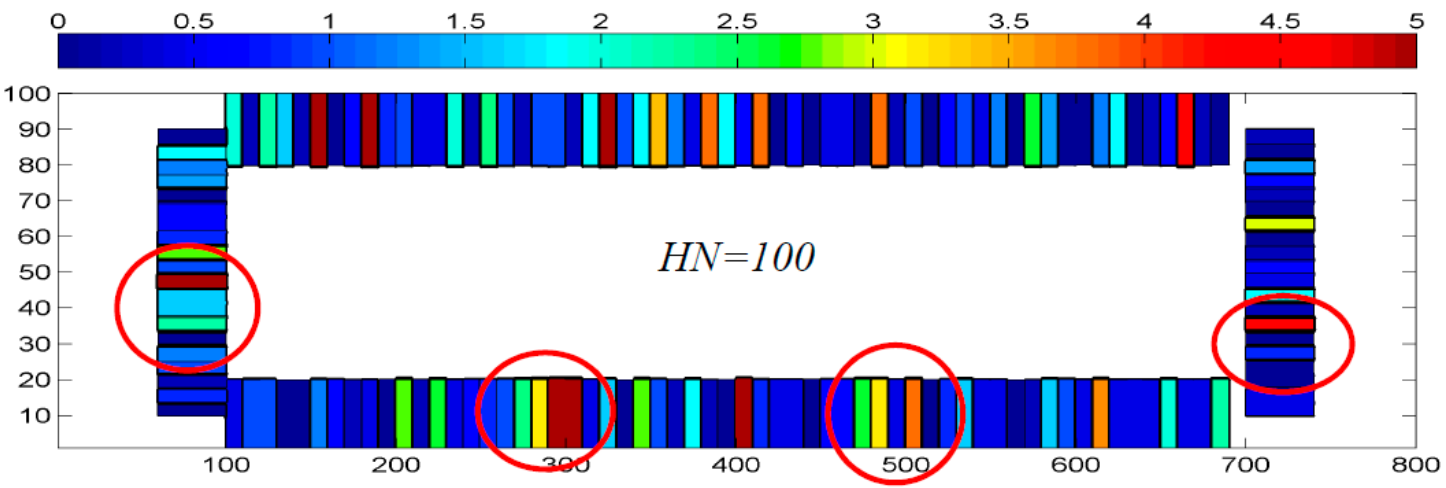

(a)

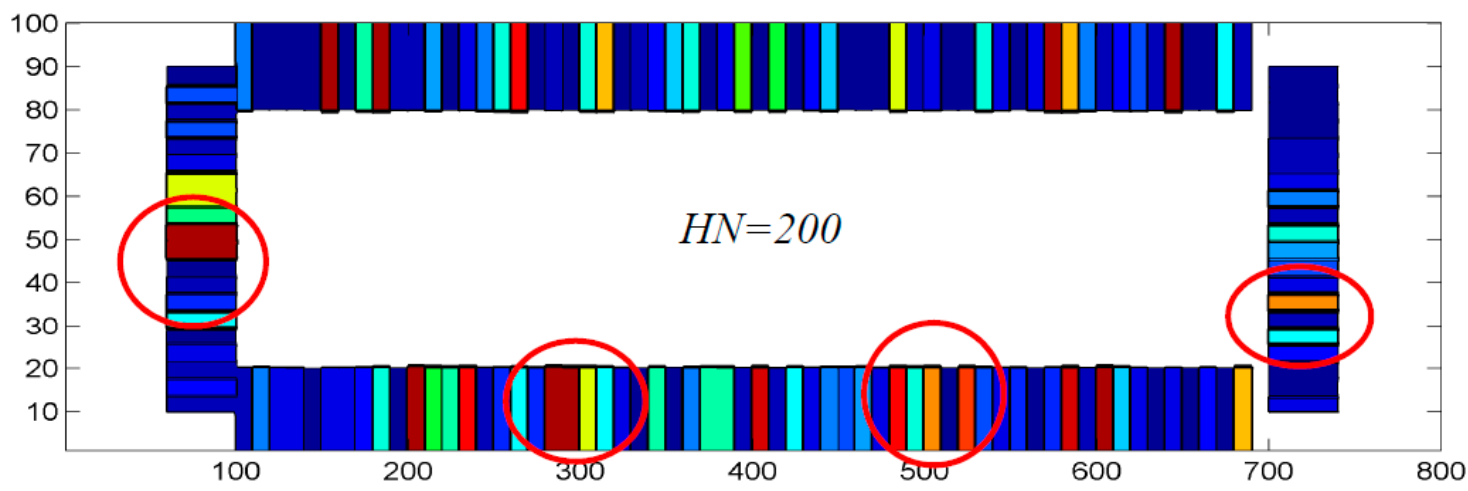

(b)

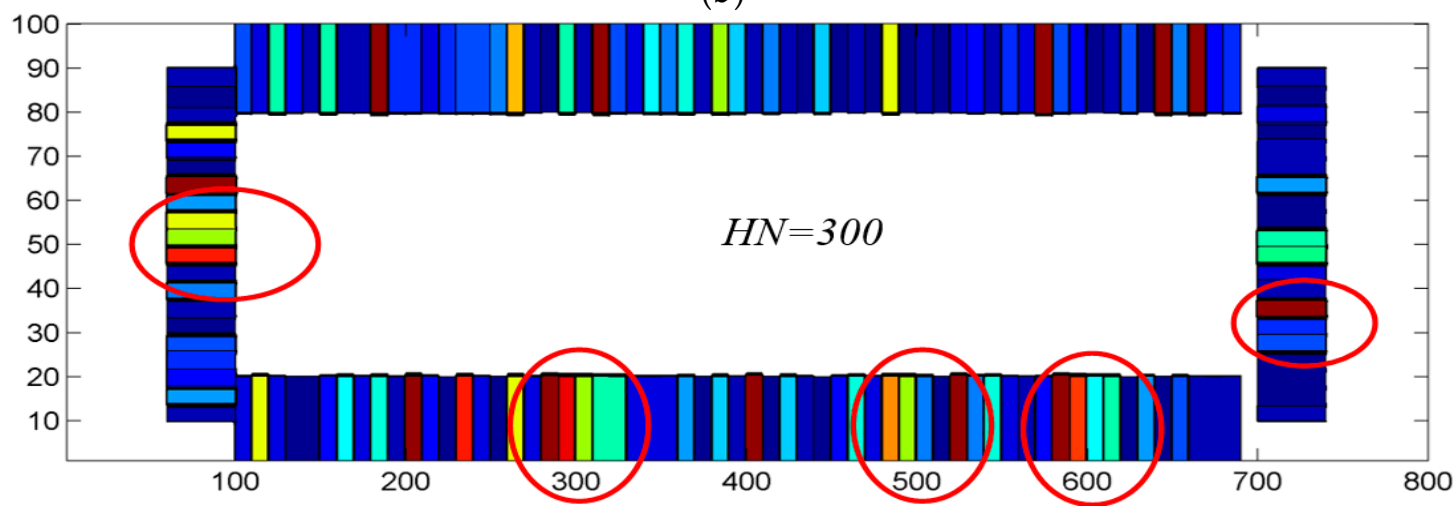

(c)

Figure 12. Accuracy of localization by using neural networks for different number of neurons in the hidden layer. (a) $H N=100 ;$ (b) $H N=200 ;$ (c) $H N=300$.

In Figure 13, accuracy of localization by using WkNN for different numbers of neighboring points is presented. 


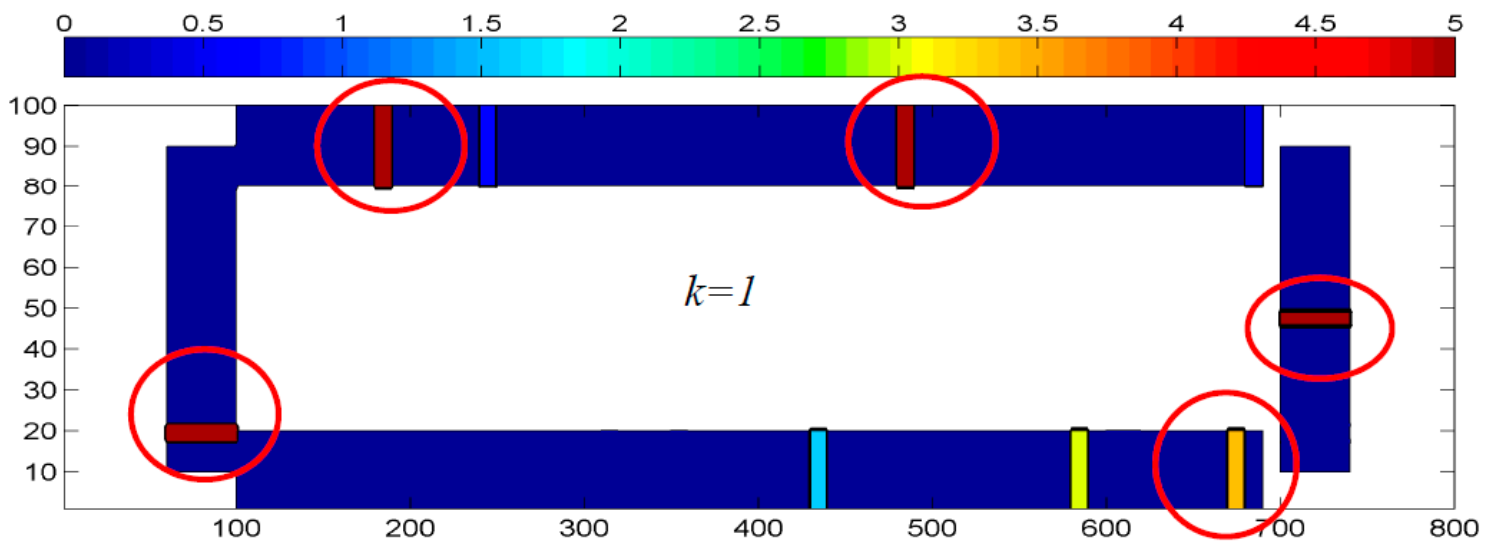

(a)

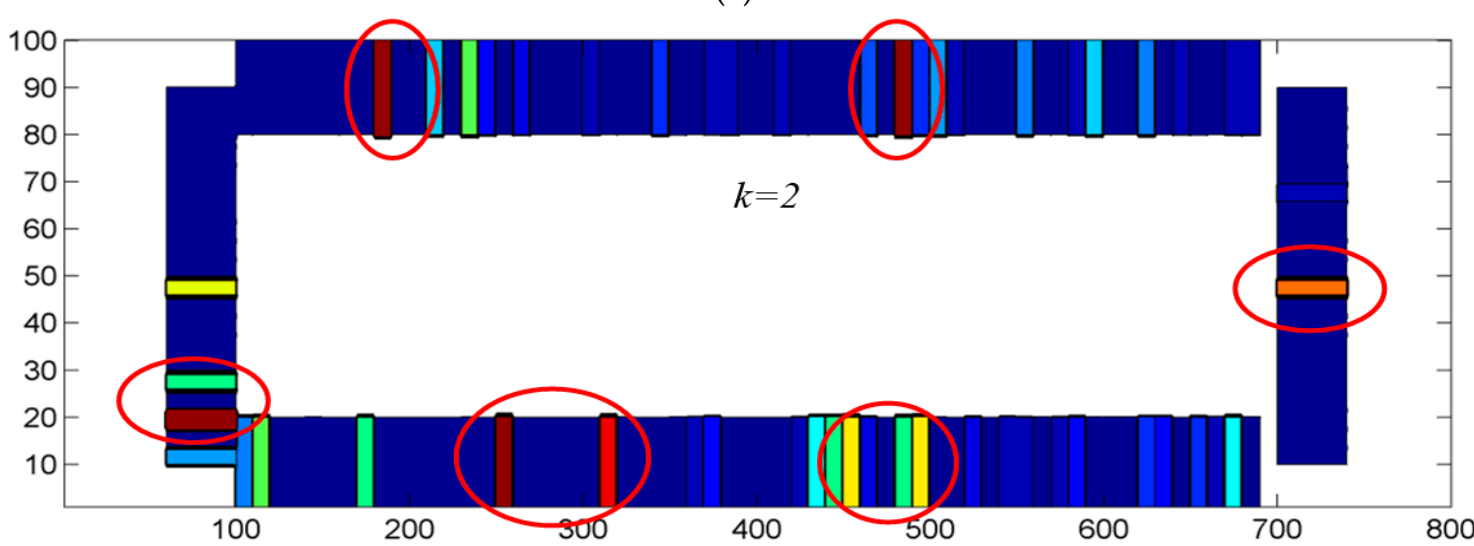

(b)

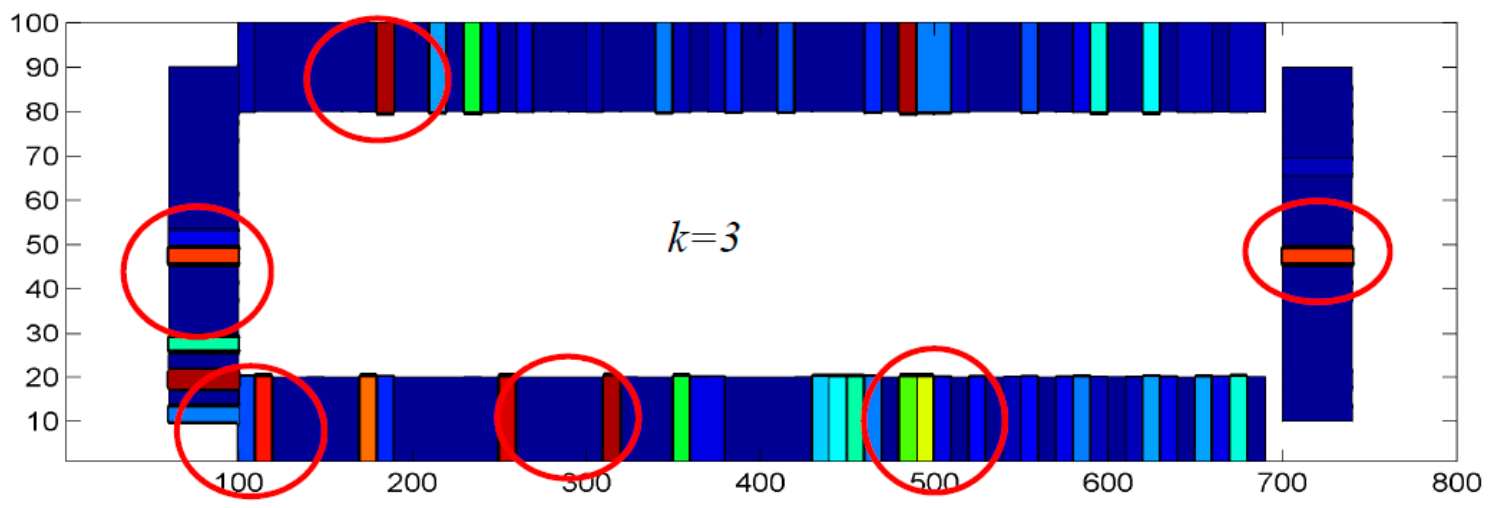

(c)

Figure 13. Accuracy of localization by using WkNN for different numbers of neighboring points. (a) $k=1$; (b) $k=2$; (c) $k=3$.

\section{Discussion}

To be able to evaluate the assembly system, i.e., to interpret the results obtained, it has to be noted, that the laboratory, as well as the assembling line itself, consists of metal elements such as tools, holders, cables, etc.; therefore, in no circumstances could a homogeneous distribution be obtained. It has to be pointed out that near Anchor 4, an industrial SCARA robot arm was located, which presented a huge metal obstacle, and therefore had a huge influence on the distribution of the RSSI values (Figure 8d). In the middle of the assembling line, Anchor 5 was positioned. The assembling line was rather narrow; therefore, the distribution of the RSSI signal in the case of Anchor 5 was rather diverse (Figure 8e).

Results presented in Figure 8 were mainly used to check whether the anchors were positioned well. Based on the presented data, one can conclude that the anchors were 
positioned well, and that the number of the anchors was satisfactory for the required coverage of the assembling line.

Based on the presented data, it can be concluded that the RSSI values obtained at the industrial laboratory are distributed more evenly than at the test laboratory [28]. This was particularly true in the case of Anchor 5. Anchor 5 was located at the center of the assembly line, and due to relatively small size of the line, the signal distribution was rather even. In the case of Anchor 5, the majority of the measurements had an RSSI value 13, while the other values (from RSSI 8 to RSSI 15 were distributed over only 15 measurement points. In the case of Anchor 4, the majority of measurements had an RSSI value of 8, 9, and 10. The most probable reason for this result is the presence of SCARA robot in that area. In the case of Anchors 1, 2 and 3, the majority of measurement results are 7, 8, 9, and 10.

The characteristic values of the anchors were almost the same. Only in the case of Anchor 5 there existed a difference. The reason for this deviation was that Anchor 5 was placed on the center of the assembly structure.

When analyzing the cumulative distribution function for quality determination in the case of localization, it can be noted that the localization effectiveness was better if the CDF curve had a greater slope. In that case, the probability of localizing the points with lower error was higher. In this case, all curves showed excellent results. The localization accuracy for this case did not depend highly on the surroundings in which the wireless technology was used. For both surroundings, higher probability of lower errors was obtained using WkNN algorithms.

In the case of the localization with "unknown" test points, the quality differed significantly compared to the quality in the case of localization with "known" test points. In this case too, the best result was obtained by using WkNN localization algorithm, with the probability of 0.5 for accuracies of $1 \mathrm{~m}$. It can be noticed that the neural network provided better results in the case of the industrial laboratory. This can be explained having in mind that the resolution in the industrial laboratory was higher (the distance between measured RSSI values was $10 \mathrm{~cm}$ ). The quality of the localization was better if $\mathrm{WkNN}$ algorithms were used and if the RSSI map was determined by measuring more points, i.e., using higher resolution.

The influence of the anchor structure to the quality of the localization yielded best results when $\mathrm{WkNN}$ algorithm with three anchors was used. For example, if the accuracy was $1.5 \mathrm{~m}$, the probability was 0.8 with three anchors compared with 0.6 for five anchors. In the case of using neural networks, no significant improvements were obtained by changing the number of anchors. Slightly better results were obtained by using five anchors.

Influence of the laboratory structure to the quality of the localization was presented in Figure 12 for NN and Figure 13 for WkNN. Based on the data in Figure 12, it can be concluded that the accuracy of the localization in certain segments always differed from the required accuracy regardless of the number of neurons. Almost all segments with high error values were also present if the localization was performed by using WkNN algorithm. By using WkNN algorithms, the performances of the localization was better, but there were segments in which the localization error differed from the majority of the assembling line.

\section{Conclusions}

The presented research proposes a supervision model customized for assembling processes, and introduces wireless sensor network technology for process management, i.e., for solving the localization problem during the process. The localization of assembled parts is based on the fingerprint localization method by using a received signal strength indicator. The proposed localization algorithms are based both on artificial neural networks and on weighted k-nearest neighbor method. The research results present a general solution to the problem of supervising the assembling process, which can be used in assembling systems regardless of the size and shape of the system.

Based on the presented data, it can be concluded that the RSSI values obtained depend on the size and shape of the system, and that a more even signal distribution can be obtained 
in the case of smaller and simpler layouts. Additionally, the density of the measured points correlates to the obtained accuracy. Obstacles, however, have a disturbing influence on the results.

Comparing the ANN and WkNN localization algorithms, better results are obtained by using WkNN algorithm. Additionally, the initial higher number of fixed anchors gave worse results than a lower number of anchors. Changing the number of neurons in the hidden layer of ANN and changing the number of neighboring points in the case of WkNN did not changed significantly the localization accuracy.

Based on the results of this analysis, the critical segments regarding the efficiency of the localization were determined. Based on this, insight was obtained regarding algorithm improvements and/or RSSI data filtering.

Author Contributions: Conceptualization: L.G.; methodology: L.G.; software: L.G.; validation: L.G. and I.F.; formal analysis: L.G. and I.F.; investigation: L.G. and I.F.; resources: L.G.; data curation: L.G. and I.F.; writing—original draft preparation: L.G. and I.F.; writing—review and editing: I.F.; visualization: L.G. and I.F. supervision: I.F.; project administration: L.G. and I.F.; funding acquisition: L.G. and I.F. All authors have read and agreed to the published version of the manuscript.

Funding: This research received no external funding.

Data Availability Statement: Data available in a publicly accessible repository.

Conflicts of Interest: The authors declare no conflict of interest.

\section{References}

1. Mareca, H. On World's 2012 Survey Shows Continued Growth and New Opportunities for Wireless Sensors. In WebExclusive, Industrial Wireless Sensor Networks Trends and Developments; InTech: London, UK, 2012.

2. Gungor, V.C.; Hancke, G.P. Industrial Wireless Sensor Networks: Challenges, Design Principles, and Technical Approaches. IEEE Trans. Ind. Electron. 2009, 56, 4258-4265. [CrossRef]

3. Vinyals, M.; Rodriguez-Aguilar, J.A.; Cerquides, J. A Survey on Sensor Networks from a Multiagent Perspective. Comput. J. 2011, 54, 455-470. [CrossRef]

4. Strasser, T.I; Armendariz, M.; Vasilenko, O.O.; Saleem, A.; Nordström, L. Multiagent-Based Distribution Automation Solution for Self-Healing Grids. IEEE Trans. Ind. Electron. 2015, 62, 2620-2628. [CrossRef]

5. Leitao, P.; Vrba, P.; Strasser, T. Multi-agent systems as automation platform for intelligent energy systems. In Proceedings of the IECON 2013-39th Annual Conference of the IEEE Industrial Electronics Society, Vienna, Austria, 10-13 November 2013; pp. 66-71.

6. Nagorny, K.; Colombo, A.W.; Schmidtmann, U. A service-and multi-agent-oriented manufacturing automation architecture: An IEC 62264 level 2 compliant implementation. Comput. Ind. 2012, 63, 813-823. [CrossRef]

7. Oborski, P. Developments in integration of advanced monitoring systems. Int. J. Adv. Manuf. Technol. 2014, 75, 1613-1632. [CrossRef]

8. Gaj, P.; Jasperneite, J.; Felser, M. Computer Communication within Industrial Distributed Environment—A Survey. IEEE Trans. Ind. Inform. 2012, 9, 182-189. [CrossRef]

9. Wollschlaeger, M.; Sauter, T.; Jasperneite, J. The Future of Industrial Communication: Automation Networks in the Era of the Internet of Things and Industry 4.0. IEEE Ind. Electron. Mag. 2017, 11, 17-27. [CrossRef]

10. Muhamad, M.D.A.; Thamrin, M.N.; Abdullah, S.A.C.; Mohamad, Z. An IoT-based production monitoring system for assembly line in manufacture. Int. J. Integr. Eng. 2020, 12, 38-45.

11. Kumar, M.; Vaishya, R. Parag Real-Time Monitoring System to Lean Manufacturing. Procedia Manuf. 2018, 20, 135-140. [CrossRef]

12. Chen, W. Intelligent manufacturing production line data monitoring system for industrial internet of things. Comput. Commun. 2020, 151, 31-41. [CrossRef]

13. Abrishambaf, R.; Bal, M.; Hashemipour, M. Distributed Control Architecture for Wireless Sensor Networks Using IEC 61499 Function Blocks for Industrial Automation. Int. J. Comput. Electr. Eng. 2014, 3, 640-644. [CrossRef]

14. Huang, G.Q.; Zhang, Y.F.; Chen, X.; Newman, S.T. RFID-enabled real-time wireless manufacturing for adaptive assembly planning and control. J. Intell. Manuf. 2008, 19, 701-713. [CrossRef]

15. Tsai, Y.S.; Chen, R.S.; Chen, Y.G.; Yeh, C.P. An RFID-based manufacture process control and supply chain management in the semiconductor industry. Int. J. Inf. Technol. Manag. 2013, 12, 85. [CrossRef]

16. Ostasevicius, V.; Jurenas, V.; Markevicius, V.; Gaidys, R.; Zilys, M.; Cepenas, M.; Kizauskiene, L. Self-powering wireless devices for cloud manufacturing applications. Int. J. Adv. Manuf. Technol. 2015, 83, 1937-1950. [CrossRef]

17. Waldemar, G. Final Results of Assembly Line Balancing Problem. In Assembly Line - Theory and Practice; InTech: London, UK, 2011; ISBN 978-953-307-995-0. 
18. Zhao, G. Wireless Sensor Networks for Industrial Process Monitoring and Control: A Survey. Netw. Protoc. Algorithms 2011, 3 , 46-63. [CrossRef]

19. Manfredi, S.; Di Tucci, E. Decentralized Control Algorithm for Fast Monitoring and Efficient Energy Consumption in Energy Harvesting Wireless Sensor Networks. IEEE Trans. Ind. Inform. 2016, 13, 1513-1520. [CrossRef]

20. Potsch, A.; Berger, A.; Springer, A. Efficient analysis of power consumption behaviour of embedded wireless IoT systems. In Proceedings of the 2017 IEEE International Instrumentation and Measurement Technology Conference (I2MTC), Turin, Italy, 22-25 May 2017; pp. 1-6.

21. Iyengar, S.S.; Brooks, R.R. Distributed Sensor Networks: Sensor Networking and Applications; CRC Press: Boca Raton, FL, USA, 2016

22. Gogolak, L. Uticaj Bežične Senzorske Tehnologije na Upravljanje Montažnim Sistemima. Ph.D. Thesis, University of Novi Sad, Novi Sad, Serbia, 2014.

23. McFarlane, D.; Giannikas, V.; Wong, A.C.; Harrison, M. Product intelligence in industrial control: Theory and practice. Annu. Rev. Control. 2013, 37, 69-88. [CrossRef]

24. Bachrach, J.; Taylor, C. Localization in sensor networks. In Handbook of Sensor Networks: Algorithms and Architectures 1; John Wiley \& Sons, Inc.: Hoboken, NJ, USA, 2005.

25. Whitehouse, K.; Karlof, C.; Culler, D. A practical evaluation of radio signal strength for ranging-based localization. ACM Sigmobile Mob. Comput. Commun. Rev. 2007, 11, 41-52. [CrossRef]

26. Gogolak, L.; Pletl, S.; Kukolj, D. Indoor fingerprint localization in WSN environment based on neural network. In Proceedings of the 2011 IEEE 9th International Symposium on Intelligent Systems and Informatics, Subotica, Serbia, 8-10 September 2011; pp. 293-296.

27. Pivato, P.; Palopoli, L.; Petri, D. Accuracy of RSS-Based Centroid Localization Algorithms in an Indoor Environment. IEEE Trans. Instrum. Meas. 2011, 60, 3451-3460. [CrossRef]

28. Gogolak, L.; Pletl, S.; Kukolj, D. Neural Network-based Indoor Localization in WSN Environments. Acta Polytech. Hung. 2013, 10, 221-235. [CrossRef]

29. Gustafsson, F.; Gunnarsson, F.; Lindgren, D. Sensor models and localization algorithms for sensor networks based on received signal strength. EURASIP J. Wirel. Commun. Netw. 2012, 2012, 16. [CrossRef]

30. Kermani, B.G.; Schiffman, S.S.; Nagle, H.T. Performance of the Levenberg-Marquardt neural network training method in electronic nose applications. Sensors Actuators B Chem. 2005, 110, 13-22. [CrossRef]

31. Kukolj, D.; Vuckovic, M.; Pletl, S. Indoor Location Fingerprinting Based on Data Reduction. In Proceedings of the 2011 International Conference on Broadband and Wireless Computing, Communication and Applications, Barcelona, Spain, 26-28 October 2011; pp. 327-332.

32. Chun, M.-H.; Han, S.-J.; Tak, N.-I. An uncertainty importance measure using a distance metric for the change in a cumulative distribution function. Reliab. Eng. Syst. Saf. 2000, 70, 313-321. [CrossRef] 